\title{
Structural-phase transformations in the zirconium single crystal under the pressure of the deformation
}

\author{
L. Yu. Egorova ${ }^{\dagger}$ Yu. V. Khlebnikova, V.P. Pilyugin, E. G. Chernyshev \\ †egorova@imp.uran.ru
}

M. N. Mikheev Institute of Metal Physics, Ural Branch of RAS, 18 S. Kovalevskoi st., Yekaterinburg, 620137, Russia

\begin{abstract}
The structural-phase transformation in pseudo-single crystal of zirconium during deformation on Bridgman anvils at room temperature with applied pressure up to $8 \mathrm{GPa}$ is studied by X-ray diffractometry with synchrotron radiation. The angles of rotation in the Bridgman anvils turning varied from $\varphi=0 \mathrm{deg}$ (deformation by draft) to $\varphi=45 \mathrm{deg}$. This method allows to evaluate the structural state of a sample throughout its thickness and to calibrate a state with a more accurate estimate of the true strain. It was shown that the $\alpha$-phase lattice is compressed at a true strain value $e=4.6$, which is reflected in its parameters: the $\alpha$-phase parameter " $\alpha$ " decreases by $5.3 \%$, and the parameter " $c$ " by $0.3 \%$. On the contrary, the $\omega$-phase arising in the process of deformation under pressure showed a tendency to stretching, if the parameter " $a$ " of this phase practically does not change with an increase in the degree of deformation, then the parameter " $c$ " is insignificant, by $0.14 \%$, but increases. This behavior can probably be explained by the small atomic density and strong anisotropy in terms of the atomic density of the planes and series of the $\omega$-phase. It is determined that the structure arising in the process of deformation of the baric $\omega$-phase, deformation conditions from $e=0.5$ to $e=4.6$, does not undergo textural changes. At this step of deformation, only the stage of nucleation of the $\omega$-phase is observed only in area of the $\alpha$-phase, which have a favorable crystallographic orientation. The formation of groups of planar defects in the $\omega$ phase is a mechanism for the compensation of elastic stresses during lattice transformation $\alpha \rightarrow \omega$ under conditions of high quasihydrostatic pressure.
\end{abstract}

Keywords: pseudo-single crystal of zirconium, deformation, high quasihydrostatic pressure, $\alpha \leftrightarrow \omega$ phase transitions.

УДК:669.295:539.89

\section{Структурно-фазовые превращения в монокристаллическом цирконии при деформации сдвигом под давлением}

\author{
Егорова Л. Ю. ${ }^{\dagger}$ Хлебникова Ю. В., Пилюгин В.П., Чернышев Е.Г. \\ Институт физики металлов имени М.Н. Михеева УрО РАН, ул. С. Ковалевской, 18, Екатеринбург, 620137, Россия
}

Методом рентгеновской дифрактометрии в синхротронном излучении на просвет проведено исследование структурно-фазового превращения в псевдомонокристаллическом $\alpha$-цирконии при деформации на наковальнях Бриджмена при комнатной температуре с приложенным давлением до 8 ГПа и угловой скоростью $\omega=0,3$ об/мин. Углы поворота наковальни варьировали от $\varphi=0^{\circ}$ (деформация осадкой) до $\varphi=45^{\circ}$. Данный метод съемки на просвет и применение малого, в сечении, пучка позволяет оценить структурное состояние образца по всей его толщине и аттестовать состояние с более точной оценкой истинной деформации. Было показано, что при величине истинной деформации $e=4,6$ решетка $\alpha$-фазы сжимается, что отражается на ее параметрах: параметр «а» $\alpha$-фазы уменьшается на 5,3\%, а параметр «с» на 0,3\%. Возникающая в процессе деформации под давлением $\omega$-фаза, наоборот, показала склонность к растягиванию, если параметр «а» данной фазы практически не меняется при увеличении степени деформации, то параметр «с» незначительно, на $0,14 \%$, но увеличивается. Такое поведение, вероятно, можно объяснить небольшой атомной плотностью и сильной анизотропией по атомной плотности плоскостей и рядов $\omega$-фазы. Определено, что структура, возникающей в процессе деформации барической $\omega$-фазы, в условиях деформирования от $e=0,5$ до $e=4,6$ не претерпевает текстурных изменений. На данном этапе деформации наблюдается лишь стадия зарождения $\omega$-фазы только в тех участках $\alpha$-фазы, которые имеют благоприятную кристаллографическую ориентацию. Образование групп планарных дефектов в $\omega$-фазе представляет собой механизм компенсации упругих напряжений при трансформации кристаллической решетки $\alpha \rightarrow \omega$ в условиях высокого квазигидростатического давления.

Ключевые слова: псевдомонокристалл циркония, деформация, высокое квазигидростатическое давление, $\alpha \leftrightarrow \omega$ фазовые переходы. 


\section{1. Введение}

Востребованность титана и циркония, а так же их сплавов в авиа- и космической отраслях, в качестве радиационностойких материалов в атомной энергетике порождает интерес к исследованию структурных превращений, которые возможны в этих металлах в разных термобарических условиях. В литературе можно встретить работы, посвященные обсуждению $\alpha \rightarrow \omega$ и $\omega \rightarrow \alpha$ превращений [1 -5]. Так, в работе [1] обсуждается вероятность диффузионной природы обратного $\omega \rightarrow \alpha$ превращения, авторы работы [2] отмечают, что при $\alpha \rightarrow \omega$ превращении в центре испытуемого образца наблюдается значительный локальный рост давления, в связи с более высоким пределом текучести $\omega$-фазы. В [5] наблюдали искажения кристаллической структуры под воздействием сдвиговой деформации, что привело к возникновению модулированной структуры $\alpha$-фазы.

На сегодняшний день детально не выяснен вопрос о начальных этапах образования и последующей эволюции метастабильной барической $\omega$-фазы, образующейся в данных металлах при сдвиге под давлением. Кроме того, немаловажным фактором для изучения эволюционных процессов, происходящих в цирконии и титане при деформации и образования $\omega$-фазы, может быть использование в эксперименте исходного материала в виде псевдомонокристалла, что исключает влияние исходных границ зерен на картину эволюции деформируемой структуры.

\section{2. Цель работы}

В работе исследованы начальные этапы деформации псевдомонокристалличекого циркония в процессе деформации сдвигом под давлением при комнатной температуре. Основные результаты получены с использованием метода рентгеновской спектроскопии с синхротронным излучением на просвет. Данное исследование позволит получить новые сведения о механизме образования барической $\omega$-фазы и спрогнозировать дальнейшую эволюцию структуры при увеличении степени деформации.

\section{3. Материал и методика эксперимента}

Для решения поставленных задач методом бестигельной электронно-лучевой зонной плавки были получены образцы исходного иодидного монокристалла $\alpha$-циркония чистотой 99,9\%. Пластическую деформацию дисковых образцов осуществляли в твердосплавных наковальнях Бриджмена при давлении 8 ГПа с угловой скоростью $\omega=0,3$ об/мин, угол поворота наковальни варьировали от $\varphi=0^{\circ}$ (деформация осадкой) до $\varphi=45^{\circ}$. Степень истинной деформации (e) рассчитывали на середине радиуса образца по формуле [6]:

$$
e_{\text {ист }}=\frac{2}{\sqrt{3}} \ln \left(1+\left(\frac{\varphi \cdot r}{h_{\text {кон }}}\right)^{2}\right)^{1 / 2}+\ln \frac{h_{0}}{h_{\text {кон }}}
$$

Структурно-фазовое состояние образцов циркония после деформации исследовали электронно-ми- кроскопически на просвет тонких фольг в микроскопе JEM-200CX, а так же рентгеновским методом в синхротронном излучении (СИ) на просвет [7] в пучке сечением $0,3 \times 0,3$ мм, длиной волны 0,03685 нм. Для регистрации дифракционной картины СИ использовался детектор Marr-345. СИ-рентгенограммы были перестроены в привычные для представления и последующей численной обработки дифрактограммы интенсивности по шкале углов $2 \theta$. По положению пиков определялись параметры решетки. Данный метод съемки на просвет и применение малого, в сечении, пучка позволяет оценить структурное состояние образца по всей его толщине и аттестовать состояние с более точной оценкой истинной деформации.

\section{4. Результаты эксперимента и их обсуждение}

В настоящей работе исследовались структурные преобразования, происходящие в псевдомонокристаллическом Zr на начальных этапах деформации в наковальнях Бриджмена - от $\varphi=0$ град (деформация осадкой) до $\varphi=45$ град, где $\varphi$ - угол поворота наковален Бриджмена при испытаниях. Ранее [8], на этом же материале, нами были проведены исследования влияния степени деформации на эволюцию структуры в образцах, подвергнутых деформации осадкой, а так же на 3, 5 и 10 оборотов в наковальнях Бриджмена. Методом просвечивающей электронной микроскопии было выявлено, что при деформации осадкой на наковальнях Бриджмена с приложенным давлением до 8 ГПа фазовое $\alpha \rightarrow \omega$ превращение происходит не во всем объеме материала, а только в областях ранее появившихся в $\alpha$-фазе деформационных двойников. Было определено, что $\omega$-фаза зарождается путем сдвига кристаллической решетки $\alpha$-фазы. Было показано, что преимущественными местами появления новой фазы являются области ориентированные таким образом, что в образующейся $\omega$-фазе плоскости залегания планарных дефектов соответствуют плоскостям двойникования $\alpha$-фазы.

Нами была предпринята попытка исследовать данное фазовое превращение методом рентгеновской спектроскопии с использованием синхротронного излучения на просвет, т. к. данный метод съемки и применение малого, в сечении, пучка позволяет оценить структурное состояние образца по всей его толщине и аттестовать состояние с более точной оценкой истинной деформации.

По полученным рентгеноструктурным данным (Рис. 1) были вычислены параметры «а» и «с» (Табл. 1) решеток $\alpha$ - и $\omega$-фазы. Особенностью синхротронного излучения является тот факт, что рентгеноструктурные данные получают на малых углах рассеяния, поэтому, несмотря на то, что метод обладает высокой точностью полученных результатов, расчеты проведены вопреки установленным рекомендациям - на малых углах, что может вносить некоторые отклонения от истинных значений в параметрах решеток исследуемых фаз.

Было выявлено, что параметр «а» решетки $\alpha$-фазы при исследуемых деформациях уменьшается незначительно, в пределах $0,2 \%$ (от $a=3,22342 \AA$ для исходного 
состояния (e=0) до $a=3,21647 \AA$ для $e=4,6)$, в то время как более длинная сторона элементарной ячейки «с» уменьшается на $5,3 \%$ (от $c=5,09486 \AA$ для исходного состояния $(e=0)$ до $c=4,82619 \AA$ при $e=4,6)$. В $\omega$-фазе при деформации наблюдается более сложная картина, параметр «a» практически не изменяется (уменьшение составило $0,0003 \%$ от $a=5,01698 \AA$ при $e=0,5$ до $a=5,01696$ при $e=4,6)$, в то время как параметр «с» не уменьшается, а увеличивается с увеличением степени деформации на $0,14 \%$ (от $c=3,11650 \AA$ при $e=0,5$ до $c=3,12094$ при $e=4,6)$. Изменение в параметрах решетки в процессе деформации отражается в смещении дифракционных пиков по шкале $2 \theta$ в сторону больших углов для $\alpha$-фазы, что свидетельствует об уменьшении параметра элементарной ячейки и в сторону меньших углов для линий $\omega$-фазы, особенно это смещение становится заметным для дальних пиков на рентгенограмме (см. Рис. 1).

Увеличение параметра решетки может свидетельствовать о совокупном влиянии растягивающих напряжений при деформационном воздействии и фазовом $\alpha \rightarrow \omega$ превращении. Известно, что пластическая деформация металлов происходит путем сдвигов наиболее прочных плотноупакованных плоскостей в направлении наиболее прочных плотноупакованных рядов, в случае гексагональной решетки это направление $<110>$. Скольжение вдоль плотноупакованных рядов отвечает наименьшему перемещению атомов в акте единичного сдвига [9].

Атомная плотность и расстояние между плоскостями исследованных фаз, рассчитанные по формулам (1), представлены в таблице (см. Табл. 2):

$$
\begin{aligned}
& \rho(001)=\frac{2}{a^{2} \sqrt{3}} ; \quad \rho(100)=\frac{2}{a c} ;- \text { атомная плотность } \\
& \rho(101)=\frac{4}{a \sqrt{3 a^{2}+4 c^{2}}} \quad \begin{array}{l}
\text { по кристаллографи } \\
\text { ским плоскостям; }
\end{array} \\
& \begin{array}{c}
r(001)=\frac{c}{2} ; \quad r(100)=\frac{a}{\sqrt{3 / 2}} ; \quad \begin{array}{c}
\text { - расстояние между } \\
r(101)=\frac{a c \sqrt{3}}{\sqrt{3 a^{2}+4 c^{2}}}
\end{array}
\end{array}
\end{aligned}
$$

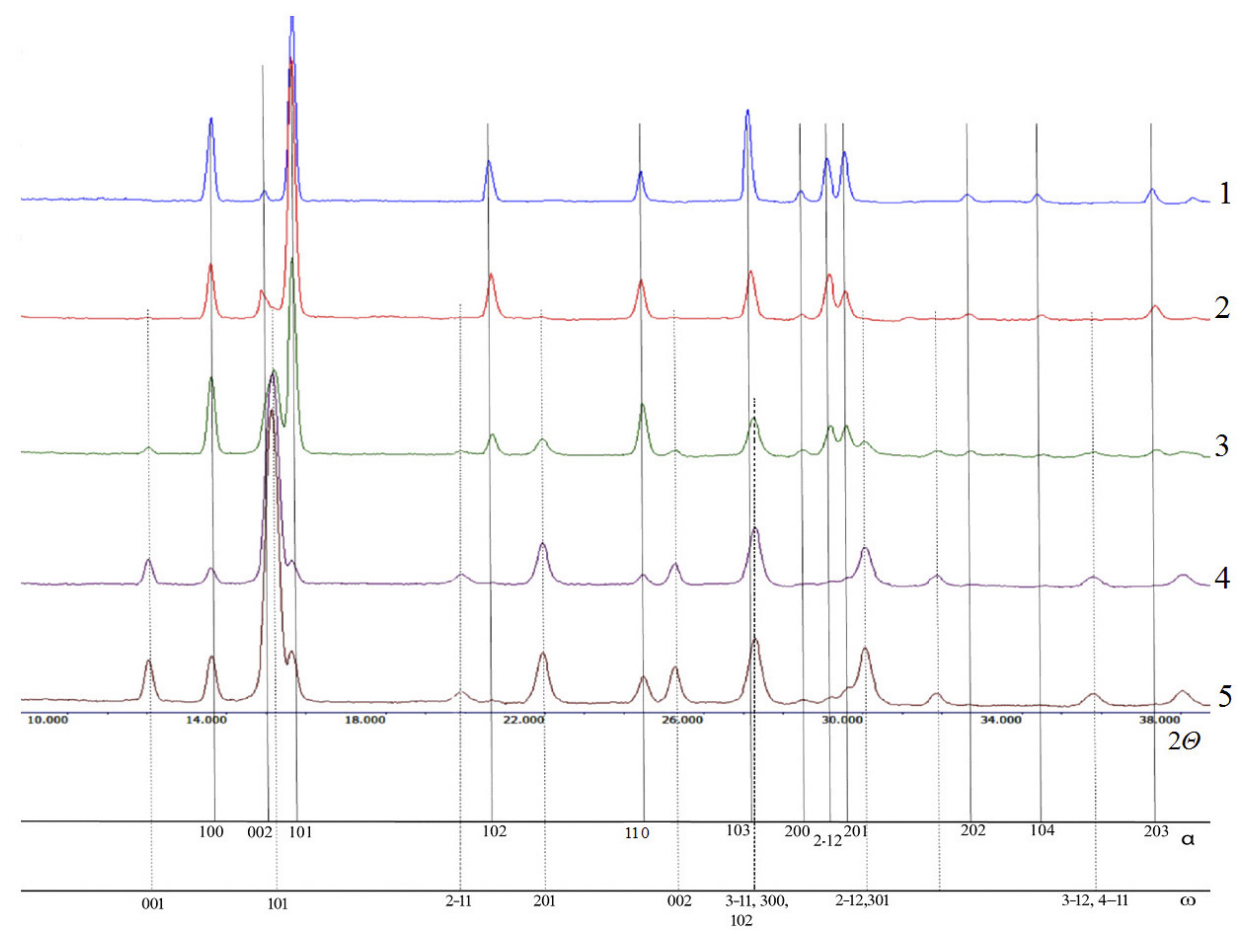

Рис. 1. Рентгеновские дифрактограммы после разной истинной деформации (е) псевдомонокристаллического циркония при $293 \mathrm{~K}$, где $1-e=0 ; 2-e=0,2 ; 3-e=0,5 ; 4-e=1,5 ; 5-e=4,6$.

Fig. 1. X-ray diffraction patterns after different true deformation $(e)$ of pseudo-single crystal of zirconium at $293 \mathrm{~K}$, where $1-e=0$; $2-e=0.2 ; 3-e=0.5 ; 4-e=1.5 ; 5-e=4.6$.

Табл. 1. Расчетные значения: истинной деформации (e); параметров решеток $\alpha$ - и $\omega$-фаз.

Table 1. Calculated values: true strain (e); parameters of lattices of $\alpha$ - and $\omega$-phases.

\begin{tabular}{|c|c|c|c|c|c|}
\hline \multirow{2}{*}{$\begin{array}{c}\text { угол поворота, град } \\
\text { rotation angle, grad }\end{array}$} & \multirow{2}{*}{$\begin{array}{c}e_{\text {ист }} \\
e_{\text {true }}\end{array}$} & $a, \AA$ & $c, \AA$ & $a, \AA$ & $c$ \\
\hline исх. / init. & 0 & 3,22342 & 5,09486 & - & - \\
\hline 0 & 0,205 & 3,22202 & 5,06558 & - & - \\
\hline 5 & 0,507 & 3,21807 & 5,04726 & 5,01698 & 3,11650 \\
\hline 15 & 1,486 & 3,21498 & 4,84302 & 5,01696 & 3,12000 \\
\hline 45 & 4,627 & 3,21647 & 4,82619 & 5,01696 & 3,12094 \\
\hline
\end{tabular}


Табл. 2. Расчетные значения атомной плотности основных плоскостей и расстояния между ними.

Table 2. Calculated values of the atomic density of the main planes and the distance between them.

\begin{tabular}{|c|c|c|c|c|c|c|}
\hline \multirow{3}{*}{$\begin{array}{l}\text { сдвиг } \varphi \text {, град. } \\
\text { shift } \varphi \text {, deg. }\end{array}$} & \multicolumn{6}{|c|}{$\alpha$} \\
\hline & \multicolumn{3}{|c|}{$\begin{array}{l}\text { атомная плотность, } \rho \\
\text { atomic density, } \rho\end{array}$} & \multicolumn{3}{|c|}{$\begin{array}{c}\text { расстояние между плоскостями, } r \\
\text { distance between planes, } r\end{array}$} \\
\hline & $\{001\}$ & $\{100\}$ & $\{101\}$ & $\{001\}$ & $\{100\}$ & $\{101\}$ \\
\hline исх. / init. & 0,11113 & 0,12178 & 0,106801 & 2,54743 & 2,631909 & 2,44816 \\
\hline 0 & 0,11123 & 0,12254 & 0,107332 & 2,53279 & 2,630768 & 2,444077 \\
\hline 5 & 0,11150 & 0,12313 & 0,107793 & 2,523629 & 2,627543 & 2,439717 \\
\hline 15 & 0,11172 & 0,12845 & 0,111359 & 2,42151 & 2,62502 & 2,413791 \\
\hline 45 & 0,11161 & 0,12884 & 0,111586 & 2,413093 & 2,626237 & 2,412537 \\
\hline \multirow{3}{*}{$\begin{array}{l}\text { сдвиг } \varphi \text {, град. } \\
\text { shift } \varphi \text {, deg. }\end{array}$} & \multicolumn{6}{|c|}{$\omega$} \\
\hline & \multicolumn{3}{|c|}{$\begin{array}{l}\text { атомная плотность, } \rho \\
\text { atomic density, } \rho\end{array}$} & \multicolumn{3}{|c|}{$\begin{array}{l}\text { расстояние между плоскостями, } r \\
\text { distance between planes, } r\end{array}$} \\
\hline & $\{001\}$ & $\{100\}$ & $\{101\}$ & $\{001\}$ & $\{100\}$ & $\{101\}$ \\
\hline исх. / init. & - & - & - & - & - & - \\
\hline 0 & - & - & - & - & - & - \\
\hline 5 & 0,04588 & 0,12792 & 0,07456 & 1,55825 & 4,096343 & 2,53240 \\
\hline 15 & 0,04588 & 0,12777 & 0,07453 & 1,56 & 4,096331 & 2,53427 \\
\hline 45 & 0,04588 & 0,12773 & 0,07452 & 1,56047 & 4,096331 & 2,53478 \\
\hline
\end{tabular}

Из этих данных видно, что атомная плотность семейства призматических плоскостей $\{100\} \omega$-фазы выше плотности базисных плоскостей $\{001\}$ в 2,8 раза, а пирамидальных $\{101\}$ в 1,7 раз в отличие от $\alpha$-фазы, в которой эти соотношения равны примерно 1,2 в обоих случаях. Самые плотноупакованные и наиболее прочные плоскости $\{100\}$ самые отдаленные друг от друга для обеих фаз. В $\omega$-фазе данное расстояние в разы больше, в отличие от других направлений. Из полученных расчетов видно, что $\omega$-фаза обладает сильной анизотропией по атомной плотности плоскостей и рядов, в то время, как атомная плотность $\alpha$-фазы более равномерно распределена по основным плоскостям и рядам.

Увеличение параметров решетки $\omega$-фазы, связано, по-видимому с тем, что атомные плотности (см. Табл. 2) основных плоскостей в $\omega$-фазе значительно ниже, чем в $\alpha$-фазе, а расстояния между рядами плоскостей $\{100\} \omega$ в 1,5 раза выше, чем в $\alpha$-фазе, т. е. металлические связи-перекрытия значительно слабее, что позволяет решетке, в процессе сжимающих нагрузок испытать растягивающие искажения [9].

Как было показано нами в работе [8], стимулом к образованию $\omega$-фазы на начальных этапах деформации циркония является наличие двойников в $\alpha$-фазе, плоскости двойникования которых соответствуют плоскостям залегания планарных дефектов в образующейся $\omega$-фазе. Участок $\omega$-фазы деформированного циркония с характерными плоскими дефектами в направлении [001] $\omega$ показан на Рис. 2. Наличие плоских (планарных) дефектов характерно для $\omega$-фазы, что уже отмечалось ранее в работах $[10,11,12]$, но связь планарных дефектов с наличием двойников в исходной $\alpha$-фазе этими авторами не анализировалась. Вероятно, в процессе деформации под давлением и фазовом превращении, в результате сдвигов плоскостей $\{100\} \omega$ в направлении [001] $\omega$ возникает периодичность, отличная от стандартной в гексагональной решетке, соответствующая расстоянию между плоскостями $\{100\} \omega$.

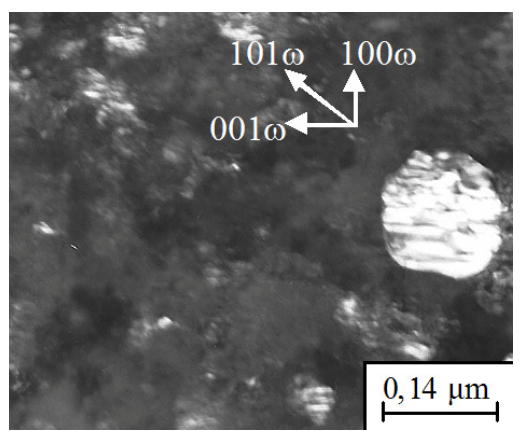

Рис. 2. Темнопольное изображение в рефлексе $(001) \omega$ структуры деформированного на $N=5$ об. $(e=8)$ псевдомонокристаллического циркония [8].

Fig. 2. The dark-field image in the reflex (001) $\omega$ of the deformed structure at $N=5$ vol. $(e=8)$ of pseudo-single crystal of zirconium [8].

Кроме смещений пиков, на рентгенограммах наблюдается и перераспределение относительных интенсивностей $\alpha$-фазы (Рис. 3). Можно наблюдать, что линия (100) $\alpha$ имеет провал интенсивности при осадке, а при деформировании в наковальнях Бриджмена с поворотом на угол $\varphi=5$ град $(e=0,5)$ интенсивность вновь возрастает. Заметное уменьшение интенсивности всех основных линий $\alpha$-фазы наблюдается при $e=1,5$ ( $\varphi=15$ град) ее значения приближаются к табличным значениям, но при деформации $e=4,6$ ( $\varphi=45$ град.) интенсивность практически всех определенных на рентгенограмме линий $\alpha$-фазы вновь возрастает. Можно предположить, что эта величина деформации соответствует смене механизма деформации: в деформационный процесс активно начинают включаться ротационные составляющие [13], что приводит к возникновению текстуры деформации и возрастанию пиков на рентгенограммах.

Подобные изменения, обусловленные текстурой деформации наблюдали в работе [6]. Здесь авторы наблюдали смену типа структуры при деформации в соответствии с изменением текстуры для металла с другим 
типом кристаллической решетки. Исследуя поведение структуры $\alpha$-железа после испытаний на наковальнях Бриджмена авторы отмечают, что повышение степени деформации на стадии ячеистой структуры сопровождается усилением относительной интенсивности линии (110) $\alpha$. Наибольшая относительная интенсивность этой линии и, соответственно, наименьшая интенсивность всех остальных отражений соответствует стадии смешанной структуры - ячеистой и сверхмелкозернистой (СМК). На стадии СМК структуры при увеличении степени деформации относительные интенсивности линий изменяются в обратной последовательности, приближаясь к значениям, характерным для бестекстурного поликристаллического железа. Авторы [6] сделали вывод, что на стадии СМК-структуры изменяется взаимная разориентировка микрокристаллитов.

При анализе результатов, полученных на псевдомонокристаллических образцах циркония, следует учитывать тот факт, что начиная с появления первых зародышей $\omega$-фазы деформации подвергается двухфазная структура. Закономерности текстурных и структурных преобразований при увеличении степени деформации для каждой из фаз могут отличаться. В отличие от $\alpha$-фазы, интенсивность дифракционных пиков, полученных от $\omega$-фазы циркония, с возрастанием степени деформации монотонно увеличивается (Рис. 4), вероятно, это происходит вследствие увеличения массовой доли барической $\omega$-фазы. Текстурное перераспределение интенсивностей линий $\omega$-фазы не наблюдается. Из этих наблюдений может следовать вывод, что появляющаяся в результате приложенного давления барическая $\omega$-фаза, при испытаниях на наковальнях Бриджмена с углом поворота наковален от $\varphi=5$ град до $\varphi=45$ град не претерпевает заметных текстурных изменений и до степени деформации $e=4,6$ ( $\varphi=45$ град) сохранятся в исходном состоянии, в независимости от направления приложенной нагрузки. Этот факт еще раз свидетельствует о том, что $\omega$-фаза возникает только при определенных кристаллографических условиях.

\section{5. Заключение}

Исследования образцов монокристаллического циркония рентгеновским методом в синхротронном излучении на просвет показали, что:

- при деформации на наковальнях Бриджмена с приложенным давлением 8 ГПа и величиной истинной деформации $e=4,6$ решетка $\alpha$-фазы сжимается, что отражается на ее параметрах: параметр «а» $\alpha$-фазы уменьшается на 5,3\%, а параметр «с» на 0,3\%. Возникающая в процессе деформации под давлением $\omega$-фаза, наоборот, показала склонность к растягиванию, если параметр «a» данной фазы практически не меняется при увеличении степени деформации, то параметр «с» незначительно, на 0,14\%, но увеличивается. Такое поведение, вероятно, можно объяснить небольшой атомной плотностью и сильной анизотропией по атомной плотности плоскостей и рядов $\omega$-фазы.

- структура, возникающей в процессе деформации барической $\omega$-фазы, в данных условиях деформирования

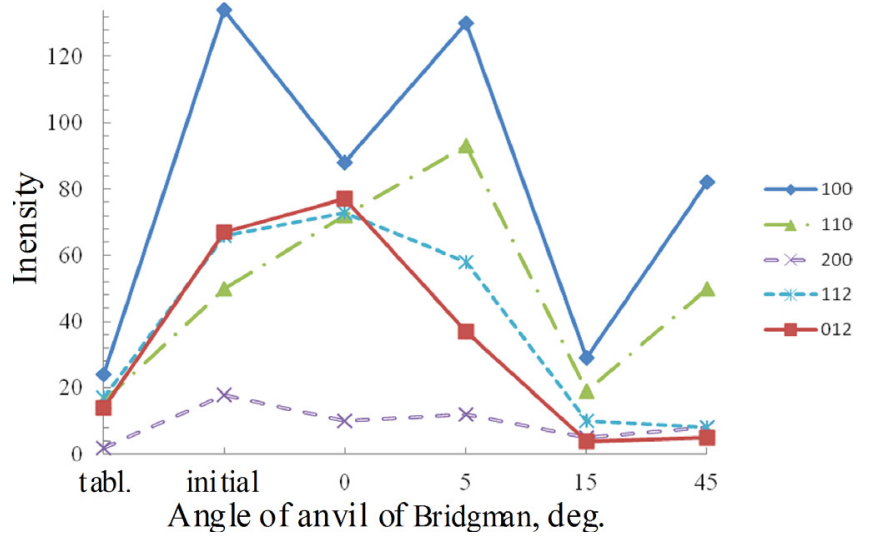

Рис. 3. Зависимость относительной интенсивности дифракционных максимумов отдельных линий $\alpha$-фазы от деформации.

Fig. 3. Dependence of the relative intensity of the diffraction maxima of the individual $\alpha$-phase lines on deformation.

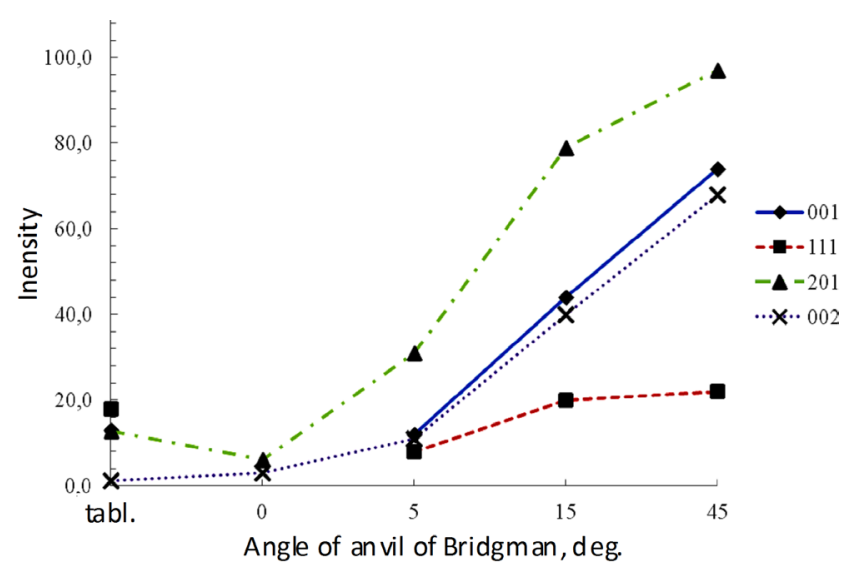

Рис. 4. Зависимость относительной интенсивности дифракционных максимумов отдельных линий $\omega$-фазы от деформации.

Fig. 4. Dependence of the relative intensity of the diffraction maxima of the individual $\omega$-phase lines on deformation.

(от $e=0,5$ до $e=4,6$ ) не претерпевает текстурных изменений. На данном этапе деформации наблюдается лишь стадия зарождения $\omega$-фазы только в участках $\alpha$-фазы, имеющих благоприятную кристаллографическую ориентацию. Образование групп планарных дефектов в $\omega$-фазе представляет собой механизм компенсации упругих напряжений при трансформации кристаллической решетки $\alpha \rightarrow \omega$ в условиях высокого квазигидростатического давления.

Благодарность/Acknowledgements. Авторы выражают благодарность А.И. Анчарову за организацию рентгеновской съемки образиов дебормированного ииркония в синхротронном излучении. Электронно-микроскопические исследования были выполнены в отделе электронной микроскопии ЦКП "Испытательный центр нанотехнологий и перспективных материалов" Института физики металлов УрО РАН. Работа выполнена в рамках государственного задания ФАНО России nо теме "Структура", №01201463331./The authors are grateful to A.I. Ancharov for organizing an $x$-ray survey of samples of deformed zirconium in synchrotron radiation. The electron microscopic studies were performed at the Center of 
the Collaborative Access "Test Center of Nanotechnologies and Advanced Materials," Institute of Metal Physics, Ural Branch, Russian Academy of Sciences. The work was carried out within the framework of the state task of the Russian Academy of Fine Arts on the topic "Structure", No. 01201463331.

\section{Литература/References}

1. N. Adachi, Yo. Todaka, H. Suzukib, M. Umemotoa. Scripta Materialia 98, 1-4 (2015). DOI: 10.1016/j. scriptamat.2014.10.029

2. B. Fenga, V. I. Levitas. Materials Science \& Engineering A. 680, 130 - 140 (2017). DOI: 10.1016/j.msea.2016.10.082

3. B. Srinivasarao, A.P. Zhilyaev, M. T. Pérez-Prado. Scripta Mater. 65 (3), 241 -244 (2011). DOI: 10.1016/j. scriptamat.2011.04.017

4. B. Srinivasarao, A. P. Zhilyaev T. G. Langdon, M. T. PérezPrado. Materials Science \& Engineering A. 562, 196- 202 (2013). DOI: 10.1016/j.msea.2012.11.027

5. X. Shen, P.F. Yu, Q. Jing, Y. Yao, L. Gu, Y.G. Wang, X. F. Duan, R. C. Yu and R.P. Liu. Scripta Materialia, 67, 653 - 656 (2012). DOI: 10.1016/j.scriptamat.2012.07.017

6. M. V. Degtyarev, L. M. Voronova, T.I. Chashchukhina, V.B. Vykhodets, L.S. Davydova, T.E. Kurennykh, A. M. Patselov, V. P. Pilyugin. The Phys. Metals Metallogr. 96 (6), $642-650$ (2003).

7. A.I. Ancharov, A.Yu. Manakov, N.A. Mezentsev, B.P. Tolochko, M.A. Sheromov, V.M. Tsukanov. Nuclear Instruments and Methods in Physics Research
Section A. 470, $80-83$ (2001). DOI: $10.1016 /$ S01689002 (01) $01029-4$

8. L. Yu. Egorova, Yu. V.Khlebnikova, V. P.Pilyugin. Letters on materials, 6, 237 - 242 (2016). (in Russian) [Егорова Л. Ю., Хлебникова Ю.В., Пилюгин В.П. Письма о материалах, 6, 237 - 242 (2016).] DOI: 10.22226/2410-3535-201 6-3-237-242

9. V.K. Grigorovich. Metal bond and the structure of metals. Moscow: Nauka (1988), 296 p. (in Russian) [Григорович В.К. Металлическая связь и структура металлов. М.: Наука (1988), 296 с.]

10. E. K. Cerreta, J.P. Escobedo, P.A. Rigg, C.P. Trujillo, D.W. Brown, T.A. Sisneros, B. Clausen, M.F. Lopez, T. Lookman, C.A. Bronkhorst, F.L. Addessio. Acta Materialia. 61, $7712-7719$ (2013). DOI: 10.1016/j. actamat.2013.09.009

11. A. V.Dobromyslov, N. I. Taluts. The Phys. Metals Metallogr. 5, 108 - 115 (1990) (In Russian) [А. В. Добромыслов, Н. И. Талуц. ФММ, 5, 108 - 115 (1990)]

12. Y. L. Alshevsky, B. A. Kulnitsky, Y. S. Konyaev, M. P. Osipov. The Phys. Metals Metallogr. 58 (4), 795-803 (1984) (in Russian) [Альшевский Ю.Л., Кульницкий Б.А., Коняев Ю.С., Усиков М.П. ФММ, 58 (4), 795-803 (1984)]

13. V.V. Rybin. Large plastic deformation and destruction of metals. M.: Metallurgy (1986), 224 p. (in Russian) [Рыбин В.В. Большие пластические деформации и разрушение металлов. М.: Металлургия (1986), 224 c.] 